Rabaska

Revue d'ethnologie de l'Amérique française

\title{
Centre de folklore acadien et créole (University of Louisiana at Lafayette)
}

\section{Barry-Jean Ancelet}

Volume 11, 2013

URI : https://id.erudit.org/iderudit/1018575ar

DOI : https://doi.org/10.7202/1018575ar

Aller au sommaire du numéro

\section{Éditeur(s)}

Société québécoise d'ethnologie

ISSN

1703-7433 (imprimé)

1916-7350 (numérique)

Découvrir la revue

Citer ce document

Ancelet, B.-J. (2013). Centre de folklore acadien et créole (University of

Louisiana at Lafayette). Rabaska, 11, 320-322. https://doi.org/10.7202/1018575ar d'utilisation que vous pouvez consulter en ligne.

https://apropos.erudit.org/fr/usagers/politique-dutilisation/ 
du Minnesota, du Dakota du Nord, du Wisconsin et de l'Iowa, avec une douzaine de professeurs de français et leurs étudiants. Le succès de cette initiative pourrait stimuler des actions scolaires et pédagogiques. La mise en place de matériel pédagogique dépend des recherches sur des sujets liés à l'héritage canadien-français. Ce terrain de recherche est riche. Depuis l'époque des explorateurs et des traiteurs des GrandsLacs, du Mississippi et des Plaines, il existe une abondance de matière. Puis s'y est ajouté le temps des pionniers qui se sont établis sur des terres, puis des ouvriers et commerçants qui se sont établis dans toutes les grandes villes et des centaines de villages. Chansons, contes, gestes et témoignages d'actes de vie quotidienne y abondent.

\section{Projets}

En voici quelques indices positifs en 2013. Une histoire de la présence canadiennefrançaise de l'État du Dakota du Nord avance vers sa publication en français. IFMidwest parraine une revue qui traite des approches à la sensibilisation de l'héritage canadien-français et michif/métis du Middle West. Le réseautage entre personnes intéressées encourage la recherche et la cueillette d'éléments historiques et folkloriques. On remarque aussi que le Middle West est en fait constitué de régions où la vie quotidienne est bien distincte. Des groupes recueillent actuellement des histoires de la région des Grands-Lacs et des Plaines. Les régions qui bordent le Mississippi possèdent bien d'autres trésors distincts. En somme, le Middle West des États-Unis s'ouvre à une nouvelle période d'exploration de son héritage et son histoire qu'il partage, en très grande partie, avec le Canada.

VIRGIL BENOIT

\section{Centre de folklore acadien et créole \\ Center for Acadian and Creole Folklore University of Louisiana at Lafayette Lafayette, LA 70504}

Téléphone : (337) 4826811

Télécopieur : (337) 4825446

Courriel: ancelet@louisiana.edu Toile : http://languages.louisiana.edu/ French/centeracadian.html

\section{Mandat et collections}

Le centre $(\mathrm{CFAC})$ continue à mettre ses collections historiques et contemporaines à la disposition du public (chercheurs, musiciens, cinéastes, enseignants, etc.), et à organiser des festivals et des spectacles spéciaux, des émissions de télévision et de radio. Il continue à dispenser des cours et des ateliers au sein des programmes universitaires, surtout en Études francophones, Histoire, Musique, et Anthropologie culturelle. Le CFAC continue à produire des documents - livres et articles, disques et vidéos - qui diffusent ces créations et ces réinterprétations auprès de la communauté et des étudiants. Il participe toujours à la production des Festivals acadiens et créoles, soit la deuxième fin de semaine d'octobre chaque année. 


\section{Projets}

Brassieur et Ancelet continuent à travailler au projet « Promised Land » pour établir un centre d'interprétation pour la culture créole à Parks.

Nous poursuivons nos travaux sur la tradition orale - contes et chansons - en Louisiane, établissant des corpus pour éventuellement examiner des traits caractéristiques dans le bagage symbolique. Ancelet, Marcia Gaudet et Ray Brassieur continuent leur documentation de l'évolution de l'architecture vernaculaire en réponse aux tempêtes et à l'érosion de la côte. Ancelet, Tamara Lindner et May Waggoner ont soumis à l'éditeur l'anthologie de la littérature d'expression française en Louisiane, des débuts de la colonie jusqu'au début du Xx siècle. Ancelet et Gaudet ont collaboré avec Carl Lindahl à la publication de Second Line Rescue : Improvised Responses to Katrina and Rita (Jackson, University Press of Mississippi). Ancelet a aussi publié Le Trou dans le mur : fabliaux cadiens (Moncton, Perce-Neige), et contribué 16 articles sur la musique cadienne et créole au Grove's Encyclopedia of Music, qui est en préparation. Gaudet continue sa direction du centre Ernest-Gaines, organisant plusieurs conférences et lectures. Brassieur continue ses recherches sur les relations entre les groupes ethniques en Louisiane, sur les plantes médicinales en Louisiane, et sur la communauté créole de La Terre Promise au long du Bayou Teche. John Laudun continue ses recherches sur la culture matérielle en Louisiane, sur les bateaux. Les membres du centre continuent à préparer une série de disques à partir de nos collections : présentement Festivals acadiens et créoles : les années 1990, et La Tradition Balfa aux Festivals acadiens et créoles. Plusieurs membres du centre ont collaboré avec le personnel du collège des beaux-arts à la réalisation d'une conférence sur Antonine Maillet et d'une performance de sa pièce Les Crasseux à Lafayette. Ancelet a aussi contribué à l'Histoire des Acadiennes et des Acadiens de la Louisiane (manuel scolaire sous la direction de Zachary Richard, Sylvain Godin, Maurice Basque, Tracadie/Sheila, Éditions La Grande Marée, 2012).

Plusieurs membres continuent leurs recherches sur le Mardi Gras en Louisiane, et participent à des interprétations de plusieurs genres (expositions, publications, festivals, etc.). Les membres du CFAC communiquent régulièrement les résultats de leurs recherches non seulement aux colloques des sociétés savantes (en histoire, linguistique, littérature, ethnologie, folklore, etc.), mais aussi dans des présentations publiques dans la région. Jennifer Ritter continue à organiser la série de présentations, « In Your Own Backyard », par lesquelles les membres de notre équipe peuvent communiquer leurs recherches au public louisianais (Veuillez visiter le site web : ccet.louisiana.edu).

\section{Projets média}

Plusieurs des membres du CFAC, dont Barry-Jean Ancelet, Ray Brassieur, Charles Richard et Connie Castille, participent à la préparation d'un documentaire sur la construction traditionnelle des bateaux. Ancelet, Bill Boelens, Chris Segura et Pat Mould ont collaboré à la production de Festivals acadiens et créoles, Live : the 1990s (Rubber Bootleg Series, Valcour Records/Center for Louisiana Studies, 2012). Ancelet, Segura et Mould ont aussi collaboré à la production de The Balfa Family 
Tradition at Festivals acadiens et créoles, 1977-2011 (Rubber Bootleg Series, Valcour Records/Center for Louisiana Studies, 2012).

\section{Activités des étudiants}

Thèses soutenues : Jean-Marie Ntipouna sur l'influence de l'ouest sur l'éducation au Cameroun); Fatoumata Modi sur la transition de l'oral à l'écrit dans la tradition de l'épopée au Niger ; Carla Gonzales-Cobos sur l'influence de la nourriture dans la littérature antillaise. Thèses en préparation : Madeline Padgett (sur la pédagogie et la technologie) ; Valerie Broussard sur la littérature et la culture cadienne en Louisiane ; Gisèle Thériault sur les histoires des pêcheurs à la baie Sainte-Marie, Nouvelle-Écosse ; Cécile Berhardt sur la littérature louisianaise du XIX ${ }^{e}$ siècle ; Laura Atran-Fresca sur l'enseignement du français. Mémoires soutenus : Maria Zéringue sur la tradition du Mardi Gras à Gheens, Louisiane ; Kory Akers sur l'évolution contemporaine de l'identité cadienne en Louisiane (en psychologie).

BARRY-JEAN ANCELET 